\title{
Salivary Gland Inverted Ductal Papilloma
}

National Cancer Institute

\section{Source}

National Cancer Institute. Salivary Gland Inverted Ductal Papilloma. NCI Thesaurus. Code C35835.

A very rare benign tumor of the minor salivary glands, characterized by a luminal papillary proliferation that arises at the junction of the salivary gland duct and the oral mucosal surface epithelium and expands as a nodular mass into the lamina propria. It presents as an asymptomatic mucosal swelling. 